\title{
Cordycepin induces apoptosis in human bladder cancer T24 cells through ROS-dependent inhibition of the PI3K/Akt signaling pathway
}

\author{
Sung Ok Kim ${ }^{1,2}$, Hee-Jae Cha ${ }^{3}$, Cheol Park ${ }^{4}$, Hyesook Lee, ${ }^{5,6}$ Su Hyun Hong ${ }^{5,6}$, \\ Soon-Jeong Jeong ${ }^{7}$, Shin-Hyung Park ${ }^{8}$, Gi-Young Kim ${ }^{9}$, Sun-Hee Leem ${ }^{10}$, Cheng-Yun Jin ${ }^{11}$, \\ Eun-Joo Hwang ${ }^{2}$, Yung Hyun Choi ${ }^{5,6, *}$
}

${ }^{1}$ Department of Food Science and Biotechnology, College of Engineering, Kyungsung University, Busan, Korea;

${ }^{2}$ Graduate School of East-West Medical Science, Kyung Hee University, Suwon, Korea;

${ }^{3}$ Department of Parasitology and Genetics, Kosin University College of Medicine, Busan, Korea;

${ }^{4}$ Department of Molecular Biology, Dong-eui University, Busan, Korea;

${ }^{5}$ Anti-Aging Research Center, Dong-eui University, Busan, Korea;

${ }^{6}$ Department of Biochemistry, Dong-eui University College of Korean Medicine, Busan, Korea;

${ }^{7}$ Department of Dental Hygiene, Youngsan University, Yangsan, Korea;

${ }^{8}$ Department of Pathology, Dong-eui University College of Korean Medicine, Busan, Korea;

${ }^{9}$ School of Marine Biomedical Sciences, Jeju National University, Jeju, Korea;

${ }^{10}$ Department of Biological Science, Dong-A University, Busan, Korea;

${ }^{11}$ School of Pharmaceutical Sciences, Zhengzhou University, Henan, China.

\begin{abstract}
Summary
Cordycepin, a derivative of nucleoside adenosine, is one of the active ingredients extracted from the fungi of genus Cordyceps, which have been used for traditional herbal remedies. In this study, we examined the effect of cordycepin on the proliferation and apoptosis of human bladder cancer T24 cells and its mechanism of action. Cordycepin treatment significantly reduced the cell survival rate of $\mathrm{T} 24$ cells in a concentration-dependent manner, which was associated with the induction of apoptosis. Cordycepin activated caspase- 8 and -9 , which are involved in the initiation of extrinsic and intrinsic apoptosis pathways, respectively, and also increased caspase- 3 activity, a typical effect caspase, subsequently leading to poly (ADP-ribose) polymerase cleavage. Additionally, cordycepin increased the $\mathrm{Bax} / \mathrm{Bcl}-2$ ratio and truncation of Bid, and destroyed the integrity of mitochondria, which contributed to the cytosolic release of cytochrome c. Moreover, cordycepin effectively inactivated the phosphoinositide 3-kinase (PI3K)/Akt signaling pathway, while LY294002, a PI3K/Akt inhibitor, increased the apoptosis-inducing effect of cordycepin. Cordycepin further enhanced the intracellular levels of reactive oxygen species (ROS), while the addition of $\mathrm{N}$-acetyl cysteine (NAC), a ROS inhibitor, significantly diminished cordycepin-induced mitochondrial dysfunction and growth inhibition, and also blocked the inactivation of PI3K/Akt signaling pathway. Furthermore, the presence of NAC significantly attenuated the enhanced apoptotic cell death and reduction of cell viability by treatment with cordycepin and LY294002. Collectively, the data indicate that cordycepin induces apoptosis through the activation of extrinsic and intrinsic apoptosis pathways and the ROS-dependent inactivation of PI3K/Akt signaling in human bladder cancer $\mathrm{T} 24$ cells.
\end{abstract}

Keywords: Cordycepin, bladder cancer, T24 cells, apoptosis, ROS, PI3K/Akt

\footnotetext{
*Address correspondence to:

Dr. Yung Hyun Choi, Department of Biochemistry, Dongeui

University College of Korean Medicine, 52-57, Yangjeong-ro,

Busanjin-gu, Busan 47227, Korea.

E-mail: choiyh@deu.ac.kr
}

\section{Introduction}

Apoptosis, a programmed cell death, is one of the most actively studied areas of cancer treatment, and 
is largely divided into death receptor (DR)-mediated extrinsic and mitochondria-mediated intrinsic pathways $(1,2)$. The extrinsic pathway begins with the activation of caspase- 8 by the formation of the death-inducing signaling complex through the binding of death ligand to the cell surface DR. On the other hand, the intrinsic pathway is characterized by the release of pro-apoptotic proteins, such as cytochrome c from the mitochondria to the cytoplasm, with increased mitochondrial permeability and the activation of caspase-9 $(1,3)$. Caspase- 8 and -9 as initiator caspases ultimately activate downstream effector caspases, including caspase-3, which induce apoptosis through the cleavage of cellular substrates. In addition, these pathways are strictly regulated by a group of proteins that are composed of pro- and anti-apoptotic proteins, such as Bcl-2 protein family proteins $(4,5)$. Meanwhile, apoptosis is precisely regulated by a wide variety of cellular signaling pathways. Among them, the phosphoinositide 3-kinase (PI3K)/Akt signaling pathway is involved in the inhibition of apoptosis and the promotion of cell growth, thus playing a key role in the pathogenesis of various tumors (6-8). Moreover, there is growing interest in reactive oxygen species (ROS), which induce apoptosis of cancer cells through dysregulation of the PI3K/Akt signaling pathway (9-11). Therefore, inhibiting the PI3K/Akt signaling pathway, while promoting the generation of ROS, can be an attractive approach to cancer treatment.

Bladder cancer is the sixth most prevalent malignancy in the United States and causes more than 16,000 deaths annually that has a considerable morbidity and mortality impact with particularly poor prognosis $(12,13)$. About $90 \%$ of affected patients are older than 55 years, and three to four times greater in men than in women to develop the disease (12). Established risk factors included male sex, older ages, personal or family history, cigarette smoking and underlying disease such as diabetes mellitus and obesity $(12,13)$. The common treatment for bladder cancer focuses on radical cystectomy, but most patients experience relapse after excision (14). Because of this, adjuvant chemotherapy is usually performed in an effort to delay recurrence and prolong survival $(14,15)$. However, there are reported that adjuvant chemotherapy responded to $50 \%$ of muscle-invasive bladder cancer patients $(15,16)$. Therefore, there is a need to develop a novel treatment strategies for the overcome these challenges of bladder cancer. In this respect, numerous medical plants and herbal pharmacologically compounds are coming into the spotlight, due to their low cost, low toxicity, and low hostility as dietary supplements (17). Recently, many studies into the natural compounds that have been traditionally used in the treatment of various diseases have shown great interest in their use as potential resources for cancer chemoprevention and chemotherapy (18-20). Among them, cordycepin is a type of nucleoside analogue that is isolated from the fungi belonging to the Cordyceps genus, such as Cordyceps militaris and C. sinensis (21-23). Although various pharmacological actions of cordycepin have been known, research on the anticancer activity, including the induction of apoptosis of cancer cells, has been conducted most extensively $(21,23-$ $25)$. For example, the intrinsic and extrinsic apoptosis pathways may be involved in the induction of apoptosis of human hepatocarcinoma and prostate cancer cells, and mouse Leydig tumor cells by cordycepin (2628). It has also been found that the increase of ROS production in leukemia, gastric and prostate cancer cells plays an important role in the induction of intrinsic apoptosis pathway (28-30). In addition, the anticancer effects of cordycepin involve the disturbance of various cell signaling pathways, and in particular, cordycepininduced apoptosis in human gastric and ovarian cancer cells and leukemia and glioma cells was accompanied by inactivation of the PI3K/Akt signaling pathway (3136). Although the induction of apoptosis by cordycepin in a certain gastric cancer cell line was accompanied by the production of ROS and inactivation of the PI3K/Akt signaling pathway (24), the underlying mechanism of ROS involved in the inactivation of $\mathrm{PI} 3 \mathrm{~K} /$ Akt signaling pathway by cordycepin is still not well known. Therefore, in this study, we investigated the effect of cordycepin on the induction of apoptosis, and evaluated whether its effect was associated with the ROS generation and PI3K/Akt signaling pathway in human urinary bladder transitional cell carcinoma T24 cells.

\section{Materials and Methods}

\subsection{Cell culture and cordycepin treatment}

T2 4 cells were purchased from the American Type Culture Collection (Manassas, MD, USA). Cells were cultured at $37^{\circ} \mathrm{C}$ in $5 \% \mathrm{CO}_{2}$ humidified incubator in complete media consisting of RPMI 1640 medium supplemented with $10 \%$ fetal bovine serum (FBS), $100 \mathrm{U} / \mathrm{mL}$ penicillin, and $100 \mu \mathrm{g} / \mathrm{mL}$ streptomycin (WelGENE Inc., Daegu, Republic of Korea). Cordycepin obtained from Sigma-Aldrich Chemical Co. (St. Louis, MO, USA) was dissolved in dimethylsulfoxide (DMSO, Sigma-Aldrich Chemical Co.) to a final concentration of $100 \mu \mathrm{g} / \mathrm{mL}$, and prior to use, the stock solution was diluted with cell culture medium to the desired concentration.

\subsection{Cell viability}

The viability of the cells was assessed by 3-(4,5-dimethyl-2-thiazolyl)-2,5-diphenyltetra-zolium bromide (MTT) assay, as previously described (37). Briefly, T24 cells were seeded onto 96-well plates at a 
density of $1 \times 10^{4}$ cells/well, and incubated overnight. Thereafter, the cells were treated with the desired concentrations of cordycepin for $48 \mathrm{~h}$, and the cells were then incubated with $50 \mu \mathrm{g} / \mathrm{mL}$ MTT solution (Invitrogen, Waltham, MA, USA) for $2 \mathrm{~h}$. Formazan crystals were dissolved in DMSO, and then the absorbance was measured by microplate reader (VERSA Max, Molecular Device Co., Sunnyvale, CA, USA) at $540 \mathrm{~nm}$. The morphological changes of cells were visualized by phase-contrast microscopy (Carl Zeiss, Oberkochen, Germany).

\subsection{Detection of apoptotic morphological changes}

Apoptotic cells containing chromatin condensation and apoptotic body formation in the nuclei were detected by $4^{\prime}, 6^{\prime}$-diamidino-2-phenylindole (DAPI, SigmaAldrich Chemical Co.) staining. After treatment with cordycepin for $48 \mathrm{~h}$, the cells were harvested, washed with phosphate-buffered saline (PBS), and then fixed with 3.7\% paraformaldehyde (Sigma-Aldrich Chemical Co.) in PBS for $10 \mathrm{~min}$ at room temperature (RT). The cells were washed with PBS, and stained with $1 \mu \mathrm{g} /$ $\mathrm{mL}$ DAPI solution for $10 \mathrm{~min}$ under light-shielded conditions. The cells were washed with PBS, and the fluorescence intensity was observed using fluorescence microscopy (Carl Zeiss).

\subsection{Determination of apoptosis by flow cytometer}

The magnitude of apoptosis was measured by flow cytometer using propidium iodide (PI) staining. In brief, the cells treated with cordycepin were washed with cold PBS, fixed in ice-cold $70 \%$ ethanol, and stored at $4^{\circ} \mathrm{C}$. The cells were suspended in cold PBS containing $50 \mu \mathrm{g} / \mathrm{mL}$ PI, $100 \mu \mathrm{g} / \mathrm{mL}$ ribonuclease A, $0.1 \%$ (w/v) sodium citrate and $0.1 \%(\mathrm{v} / \mathrm{v})$ Nonidet-P40 (SigmaAldrich Chemical Co.), then incubated on ice for 30 min in the dark at RT. Flow cytometric analysis was carried out using a flow cytometer (BD Biosciences, San Jose, CA, USA), and Cell Quest software was used to determine the relative DNA content. The sub-G1 population was calculated to estimate the apoptotic cell population.

\subsection{Agarose gel electrophoresis for DNA fragmentation assay}

After treatment with cordycepin for $48 \mathrm{~h}$, the cells were lysed in lysis buffer containing $10 \mathrm{mM}$ Tris- $\mathrm{HCl}(\mathrm{pH}$ 7.4), $150 \mathrm{mM} \mathrm{NaCl}, 5 \mathrm{mM}$ ethylenediaminetetraacetic acid, and $0.5 \%$ Triton X-100 for $1 \mathrm{~h}$ at RT. The lysates were vortexed, and cleared by centrifugation at $10,000 \times$ $\mathrm{g}$ for $30 \mathrm{~min}$. After extraction of fragmented DNA in the supernatant using phenol:chloroform:isoamyl alcohol (25:24:1, v/v/v, Sigma-Aldrich Chemical Co.), electrophoretic analysis was performed on $1.0 \%$ agarose gels containing $0.1 \mu \mathrm{g} / \mathrm{mL}$ ethidium bromide (EtBr, Sigma-Aldrich Chemical Co.).

\subsection{Protein extraction and Western blot analysis}

Total protein was extracted from the cells using the Bradford Protein assay kit (Bio-Rad Laboratories, Hercules, CA, USA), according to the manufacturer's protocol. NE-PER nuclear and cytoplasmic extraction reagents (Thermo Fisher Scientific Inc., Waltham, Utah, USA) were applied for the preparation of mitochondrial and cytosolic extracts of cells, according to the manufacturer's instructions. After quantification of protein concentration using the Bio-Rad protein assay kit (Bio-Rad Laboratories, Hercules, CA, USA), an equal amount of protein from the samples was separated by denaturing sodium dodecyl sulfate (SDS)polyacrylamide gel electrophoresis, and transferred onto polyvinylidene difluoride (PVDF) membranes (Schleicher \& Schuell, Keene, NH, USA). The membranes were blocked with $5 \%$ skim milk in Trisbuffered saline containing $0.1 \%$ Triton X-100 (TBST) for $1 \mathrm{~h}$, and probed with specific primary antibodies at $4^{\circ} \mathrm{C}$ overnight. After washing three times with TBST, the membranes were incubated with the appropriate horseradish peroxidase (HRP)-conjugated secondary antibodies for $2 \mathrm{~h}$ at RT. The expression of protein was detected by enhanced chemiluminescence (ECL) kit (GE Healthcare Life Sciences, Little Chalfont, UK), and visualized by Fusion FX Image system (Vilber Lourmat, Torcy, France).

\subsection{Caspase activity}

The activity of caspases was measured using caspase activity assay kits (R\&D Systems, Minneapolis, MN, USA), according to the manufacturer's protocol. In brief, cells were harvested, and lysed in the lysis buffer provided in the kit. The supernatants were collected, incubated with the supplied reaction buffer containing dithiothreitol, with or without substrates [Asp-Glu-ValAsp (DEAD) for caspase-3; Ile-Glu- Thr-Asp (IETD) for caspase-8; and Leu-Glu-His-Asp (LEHD) for caspase-9] labeled with p-nitroaniline (pNA) at $37^{\circ} \mathrm{C}$ for $2 \mathrm{~h}$ in the dark. The optical density of the reaction mixture was determined by absorbance at $405 \mathrm{~nm}$ using a microplate reader.

\subsection{Measurement of mitochondrial membrane potential (MMP, $\Delta \psi m)$}

To observe the changes of MMP, 5,5',6,6'-tetrachloro1,1',3,3'-tetraethyl-imidacarbocyanine iodide (JC-1; Sigma-Aldrich Chemical Co.) staining was performed. After treatment with various concentrations of cordycepin, $10 \mu \mathrm{M} \mathrm{JC}-1$ was added to the cells for 30 $\min$ at $37^{\circ} \mathrm{C}$. Subsequently, the cells were washed with 
PBS to remove unbound dye, and at least 10,000 cells were collected for each sample. The amounts of MMP were detected at $488 / 575 \mathrm{~nm}$ using a flow cytometer (BD Biosciences), by following the manufacturer's protocol.

\subsection{Statistical analysis}

The results of quantitative studies are reported as mean \pm standard deviation (SD) using GraphPad Prism software (version 5.03; GraphPad Software, Inc., La Jolla, CA, USA). All experiments were repeated at least three times. To compare data, One-way analysis of variance (ANOVA) with Dunnett's post-hoc test was used, and $p<0.05$ was considered to indicate a statistically significant difference.

\section{Results}

\subsection{Cordycepin inhibits T24 cell viability}

In evaluate the cytotoxicity of cordycepin in T24 cells, the cells were incubated with different concentrations of cordycepin for $48 \mathrm{~h}$, and cell viability was assessed by MTT assay. Figure 1A shows that cordycepin significantly reduced T24 cells viability in a concentration-dependent manner, and fifty percent inhibitory concentration $\left(\mathrm{IC}_{50}\right)$ values of cordycepin was $41.62 \mu \mathrm{M}$. Therfore, we decided that $40 \mu \mathrm{M}$ $\left(\mathrm{IC}_{50}\right)$ appropriated as the maximum concentration for investigation of apoptotic effects of cordycepin. Under phase-contrast microscope, the phenotypic characteristics of cordycepin-treated cells showed

A)

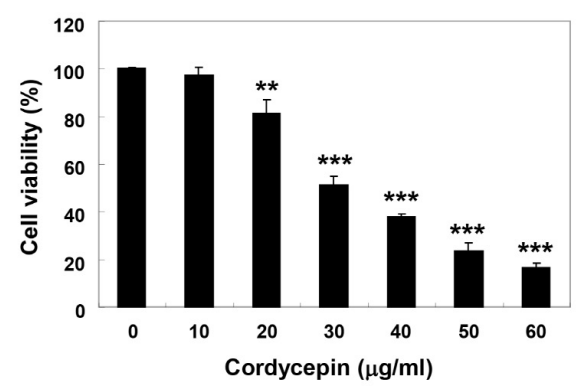

B)

\begin{tabular}{|c|c|c|c|c|}
\hline 0 & 10 & 20 & 30 & 40 \\
\hline & & & $s$ & 8 \\
\hline
\end{tabular}

Figure 1. Cordycepin decreases the survival of human bladder cancer T24 cells. (A) T24 cells were treated with the indicated concentrations of cordycepin. The cell viability was assessed after $48 \mathrm{~h}$ by MTT assay, as described in the Materials and Methods. Each bar represents the mean \pm SD of three independent experiments $(* * p<0.001$ and $* * * p<0.0001$ when compared to control). (B) Morphological changes of T24 cells were observed by phase-contrast microscopy. Representative photographs of the morphological changes are presented. irregular cell outlines, decrease of cell density, and increase of detached cell (Figure 1B).

\subsection{Cordycepin induces apoptotic cell death in T24 cells}

We determined whether the growth inhibition of T24 cells by cordycepin was associated with apoptosis induction. Figure $2 \mathrm{~A}$ shows the results of DAPI staining, which reveal that the morphological changes of nuclei observed in apoptosis-inducing cells, such as nuclear fragmentation, and chromatin condensation, were increased, depending on cordycepin treatment concentration. In addition, the results of flow cytometry analysis and agarose electrophoresis showed that the percentage of sub-G1 cells and fragmentation of DNA were increased in cells treated with cordycepin in a concentration-dependent manner (Figure 2B-D).

\subsection{Cordycepin activates caspases in T24 cells}

We next assessed whether cordycepin activated the caspase signaling pathway in T24 cells, and found that cordycepin reduced the expression of pro-caspase-3, -8 , and -9 (Figure 3A) and increased their enzymatic activity in a concentration-dependent manner (Figure 3B). Cordycepin also induced cleavage of poly (ADPribose) polymerase (PARP), one of the major substrate

A)

A) Cordycepin $(\mu \mathrm{g} / \mathrm{ml})$

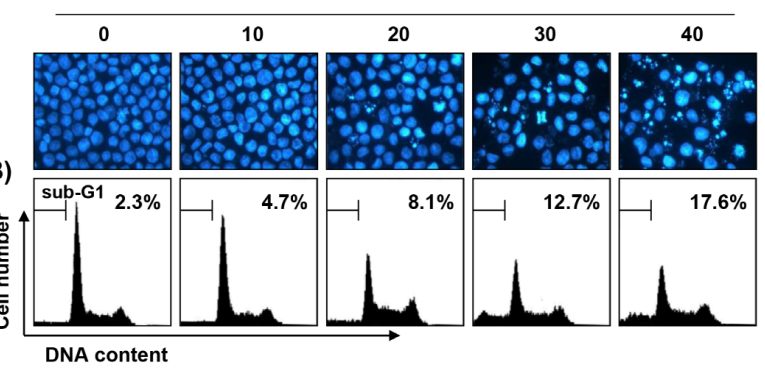

C)

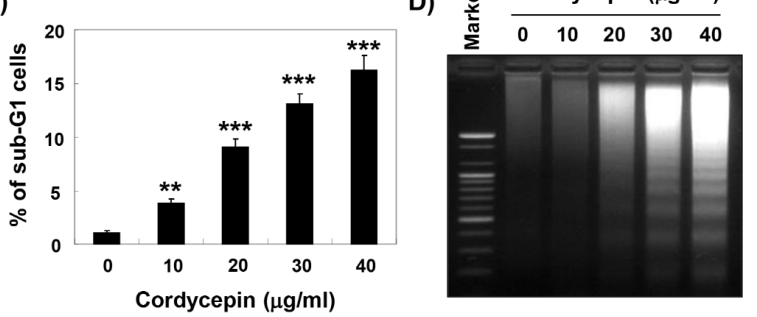

Figure 2. Cordycepin induces apoptosis in T24 cells. (A) After treatment with different concentrations of cordycepin for $48 \mathrm{~h}$, the cells were collected, fixed, and stained with DAPI solution. The stained nuclei were pictured under a fluorescence microscope. (B and C) The cells cultured under the same conditions were collected, and stained with PI solution for flow cytometry analysis. (B) The percentages of apoptotic sub-G1 cells were determined. (C) Data were expressed as the mean $\pm \mathrm{SD}$ of three independent experiments $(* * p<0.001$ and $* * * p<0.0001$ when compared to control). (D) DNA fragmentation was analyzed by the extraction of genomic DNA, electrophoresis in agarose gel, and then visualization by EtBr staining. 
A)

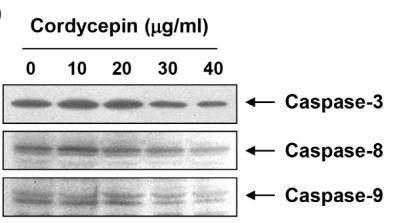

B)

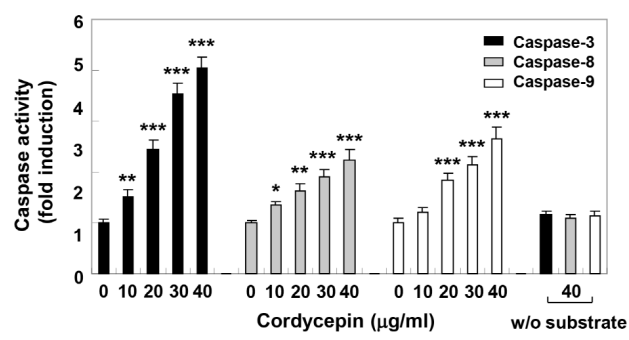

Figure 3. Cordycepin induces the activation of caspases and degradation of PARP in T24 cells. T24 cells were treated with the indicated concentrations of cordycepin for $48 \mathrm{~h}$. (A) The cell lysates were prepared, and equal amounts of cellular proteins were separated on SDS-polyacrylamide gels, and transferred to PVDF membranes. The membranes were probed with the indicated antibodies, and the proteins were visualized using an ECL detection system. The equivalent loading of proteins in each well was confirmed by actin. (B) The activities of caspases were evaluated using caspases colorimetric assay kits. The data were expressed as the mean \pm SD of three independent experiments $(* p<0.05, * * p<0.001$, and $* * * p<$ 0.0001 , when compared to control).

proteins of activated caspase-3 (Figure 3A).

\subsection{Cordycepin induces mitochondrial dysfunction in T24 Cells}

We further assessed whether mitochondrial dysfunction was involved in the induction of cordycepin-induced apoptosis, in order to study additional mechanisms involved in inducing apoptosis by cordycepin. As can be seen from the results of $\mathrm{JC}-1$ staining, the MMP-dependent formation of JC-1 aggregates in mitochondria was maintained at a relatively high rate in T24 cells not treated with cordycepin (Figure 4A and $\mathrm{B}$ ). However, JC-1 aggregates were reduced after treatment with cordycepin in a concentration-dependent manner, indicating a significant depletion of MMP after cordycepin treatment. As indicated in Figure 4C, we also found that cordycepin increased the expression of pro-apoptotic Bax, and decreased the expression of anti-apoptotic Bcl-2. Additionally, the expression of truncated $\mathrm{BH} 3$ interacting-domain death agonist (tBid) was increased, and the release of cytochrome $\mathrm{c}$ from the mitochondria to the cytoplasm was promoted in cordycepin-treated T24 cells (Figure 4C and D).

\subsection{Cordycepin inactivates PI3K/Akt signaling pathway in T24 cells}

To determine the effect of cordycepin on the PI3K/Akt signaling pathway, we measured the phosphorylation level of PI3K protein and its downstream component Akt. Figure 5A shows that when cells were exposed to

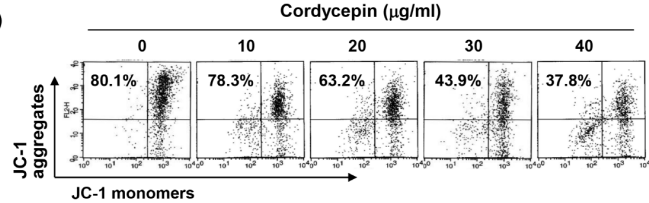

B)

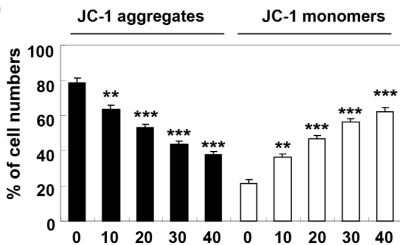

C) Cordycepin $(\mu \mathrm{g} / \mathrm{ml})$

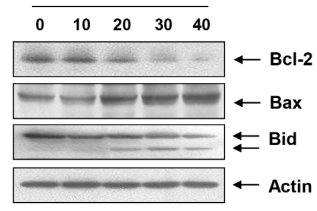

Cordycepin $(\mu \mathrm{g} / \mathrm{ml})$

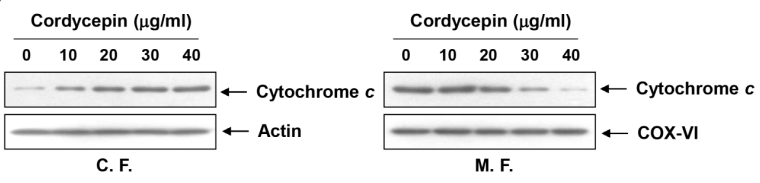

Figure 4. Cordycepin reduces the values of MMP ( $\Delta \psi \mathrm{m})$, modulates the levels of $\mathrm{Bcl}-2$ family proteins, and induces the cytosolic release of cytochrome $\mathrm{c}$ in $\mathrm{T} 24$ cells. (A) After $48 \mathrm{~h}$ incubation with the indicated concentrations of cordycepin, the cells were stained with JC-1 dye, and were then analyzed by flow cytometry, in order to evaluate the changes in MMP. (B) Each bar represents the percentage of cells with JC- 1 aggregates and monomers (mean \pm SD of triplicate determinations, $* * p<0.001$ and $* * * p 0.0001$, when compared to control). (C) The cell lysates were prepared, and the expression of $\mathrm{Bcl}-2$ family proteins $(\mathrm{Bc}-2, \mathrm{Bax}$ and Bid) was evaluated by Western blot analysis with whole cell lysates. Equal protein loading was confirmed by an analysis of actin. (D) Cytosolic and mitochondrial proteins were prepared, and analyzed for cytochrome c expression by Western blot analysis. Equal protein loading was confirmed by the analysis of actin and cytochrome oxidase subunit VI (COX VI) in each protein extract. The results shown are representative of three independent experiments.

cordycepin, the expressions of phosphorylated (p)-PI3K and $\mathrm{p}$-Akt were gradually decreased with increasing time of cordycepin treatment, while total PI3K and Akt protein levels remained constant during cordycepin treatment, which suggests that cordycepin was able to block the activation of the PI3K/Akt pathway in T2 4 cells. To further confirm the role of the PI3K/Akt pathway in cordycepin-mediated apoptosis, cells were co-treated with LY294002, a specific PI3K inhibitor and cordycepin. The results obtained from DAPI staining, agarose gel electrophoresis, and flow cytometric analysis showed that apoptosis was significantly increased in cells treated with PI3K inhibitor and cordycepin, compared to with cordycepin alone (Figure 5B-E). In addition, after co-treatment with LY294002 and cordycepin, the reduction of cell viability by cordycepin was further reduced (Figure 5F).

\subsection{Cordycepin induces ROS-dependent mitochondrial dysfunction and growth inhibition in T24 cells}

We next investigated whether cordycepin induced the production of ROS, and the effects of increased ROS on cordycepin-induced apoptosis and inhibition of the PI3K/Akt signaling pathway. Examining the 
A)

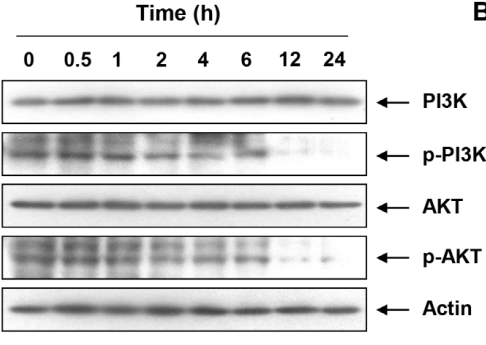

D) Cordycepin $(40 \mu \mathrm{g} / \mathrm{ml})$

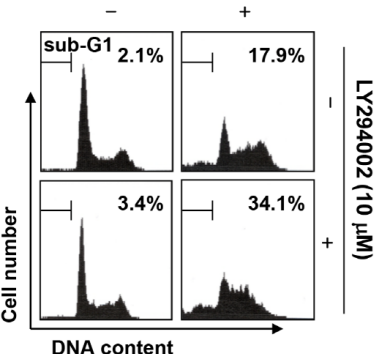

B)

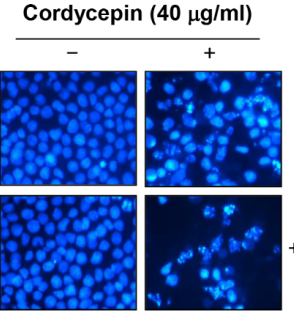

E)

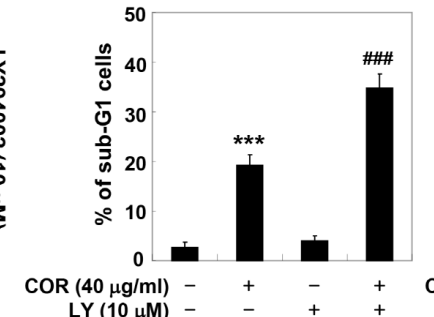

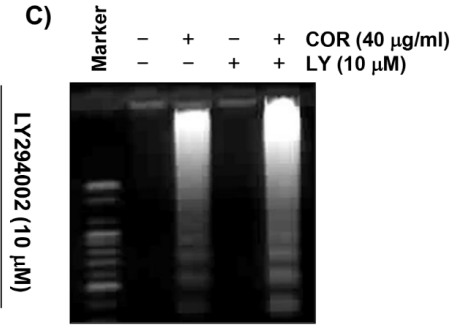

F)

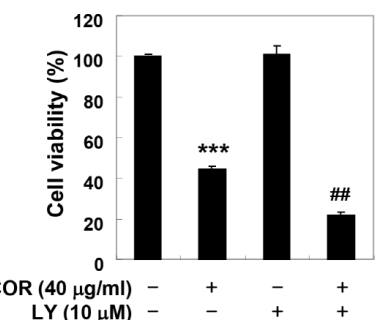

Figure 5. Cordycepin induces the inactivation of PI3K/Akt signaling pathway in T24 cells. The cells were treated with $40 \mu \mathrm{g} /$ $\mathrm{mL}$ cordycepin for the indicated times (A), or pre-treated with $10 \mu \mathrm{M} \mathrm{LY} 294002 \mathrm{for} 1 \mathrm{~h}$, and then treated with $40 \mu \mathrm{g} / \mathrm{mL}$ cordycepin for a further $48 \mathrm{~h}$ (B-F). (A) The cell lysates were prepared, and the expression of PI3K and Akt proteins was evaluated by Western blot analysis with whole cell lysates. Actin was used as an internal control. (B) The DAPI-stained nuclei were then observed by fluorescence microscopy (original magnification, $\times 400$ ). (C) DNA fragmentation was analyzed by the extraction of genomic DNA, electrophoresis in agarose gel, and then visualization by EtBr staining. (D) The percentages of apoptotic sub-G1 cells were determined. (E) Data were expressed as the mean $\pm \mathrm{SD}$ of three independent experiments $(* * * p<0.0001$, when compared to control; ${ }^{\# \#} p<0.001$, when compared to cordycepin-treated cells). (F) The cell viability was measured by MTT assay. Data were expressed as the mean $\pm \mathrm{SD}$ of three independent experiments $(* * * p<0.0001$, when compared to control; \# $p<0.001$, when compared to cordycepin-treated cells). COR, cordycepin; LY, LY294002.

generation of ROS using 2',7'-dichlorofluorescin diacetate (DCF-DA) showed that the accumulation of ROS was highest after $1 \mathrm{~h}$ of cordycepin treatment, and then gradually decreased thereafter (data not shown). However, cells co-treated with $\mathrm{N}$-acetyl cysteine (NAC), a potent ROS scavenger, showed significantly reduced ROS levels, compared to those of cordycepin alone treated cells (Figure 6A and B). Consistent with these flow cytometric results, fluorescence microscopy observations confirmed that NAC treatment significantly inhibited cordycepin-induced ROS generation (Figure 6C). In addition, NAC significantly prevented cordycepin-induced loss of MMP (Figure 6D and E), and reduced viability in T24 cells (Figure 6F).

\subsection{Cordycepin-mediated inactivation of PI3K/Akt signaling is ROS-dependent in T24 cells}

We further investigated the role of PI3K/Akt signaling pathway on ROS generation-mediated apoptosis by cordycepin. Figure 7A shows that when the production of ROS was artificially blocked, the reduced phosphorylation levels of PI3K and Akt by cordycepin were maintained at the control level. In addition, NAC treatment significantly protected the apoptosis induced by the co-treatment of cordycepin and LY294002, as observed by the nuclear morphological changes, DNA fragmentation assay, and flow cytometric analysis (Figure 7B-E). Consistent with these results, the reduced cell viability by co-treatment with cordycepin and LY 294002 was also significantly restored by blocking ROS production (Figure 7F).

\section{Discussion}

Many previous studies have shown that cordycepin induces apoptosis in a variety of cancer cells under conditions that are not toxic to normal cells $(21,23,38)$. It has also been reported that several cellular signaling pathways are involved in the induction of apoptosis of cancer cells by cordycepin $(27,32,35,38-40)$, and that the accumulation of ROS associated with mitochondrial dysfunction acts as a major signal in this process $(27,29$ $30,32,39,41)$. Although the possibility of involvement of PI3K/Akt signaling pathway was suggested in several previous studies $(32-36,42)$, the link between this pathway and the production of ROS is largely unknown.

Our results indicated that cordycepin activated both caspase- 8 and -9 , initiator caspases for the activation of extrinsic and intrinsic pathways, respectively, and increased the truncation of Bid in T24 cells, consistent with previous studies in breast and prostate cancer cells $(28,42,43)$. In addition, mitochondrial dysfunction was induced as confirmed by the loss of MMP in cordycepin-treated T24 cells. The loss of MMP was accompanied by a down-regulation in the Bcl-2/Bax ratio and promotion of cytochrome $\mathrm{c}$ release into the cytoplasm from mitochondria, which are typically 
A)

Cordycepin $(40 \mu \mathrm{g} / \mathrm{ml})$

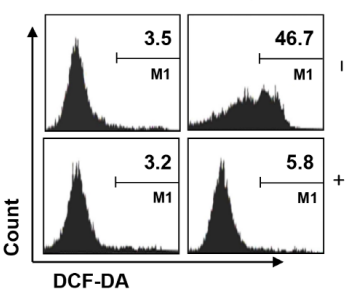

D)

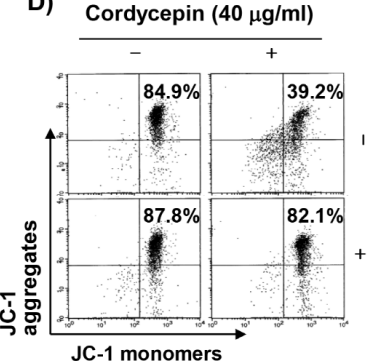

B)

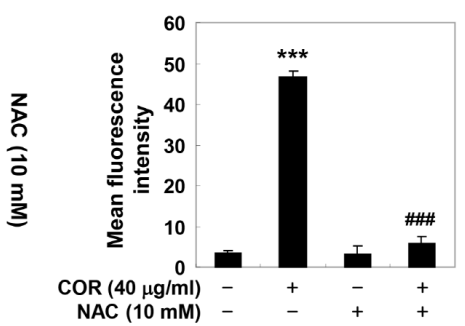

E)

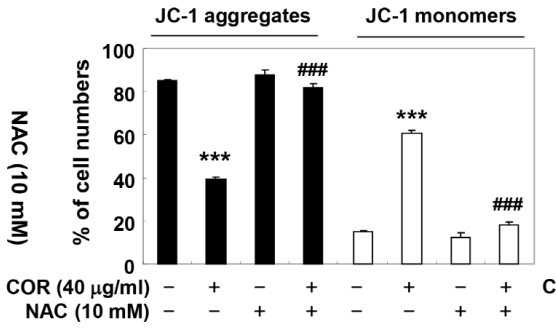

C)

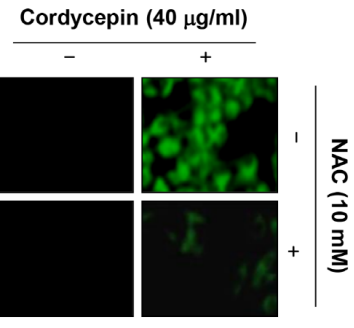

F)

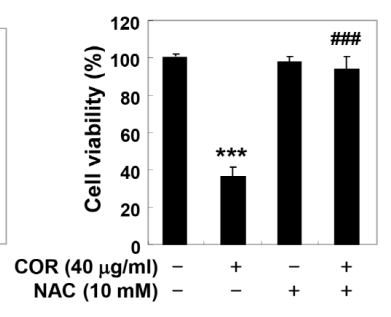

Figure 6. Cordycepin induces ROS generation in T24 cells. (A-C) The cells were either treated with $40 \mu \mathrm{g} / \mathrm{mL}$ cordycepin for $1 \mathrm{~h}$, or pre-treated with $10 \mathrm{mM} \mathrm{NAC}$ for $1 \mathrm{~h}$, before cordycepin treatment. (A) The medium was discarded, and the cells were incubated at $37^{\circ} \mathrm{C}$ in the dark for $20 \mathrm{~min}$ with new culture medium containing DCF-DA. ROS generation was measured by flow cytometry. (B) Each bar represents the mean $\pm \mathrm{SD}$ of three independent experiments $(* * * p<0.0001$, when compared to control; \#\#\# $p<0.0001$, when compared to cordycepin-treated cells). (C) Images were obtained by fluorescence microscopy (original magnification: $\times 200$ ). The images presented here are captured from one experiment, and are representative of at least three independent experiments. (DF) The cells were either treated with $40 \mu \mathrm{g} / \mathrm{mL}$ cordycepin for $48 \mathrm{~h}$, or pre-treated with $10 \mathrm{mM}$ NAC for $1 \mathrm{~h}$, before cordycepin treatment. (D) The cells were stained with JC-1 dye, and were then analyzed by flow cytometry, in order to evaluate the changes in MMP. (E) Each bar represents the percentage of cells with JC-1 aggregates and monomers (mean \pm SD of triplicate determinations, ${ }^{* * *} p<0.0001$, when compared to control; \#\# $p<0.0001$, when compared to cordycepin-treated cells). (F) The cell viability was measured by MTT assay. Data were expressed as the mean \pm SD of three independent experiments $(* * * p<0.0001$, when compared to control; ${ }^{\# \# p}<0.0001$, when compared to cordycepin-treated cells). COR, cordycepin.

A)

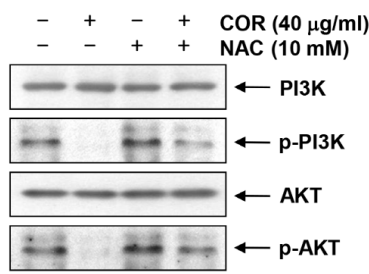

D)
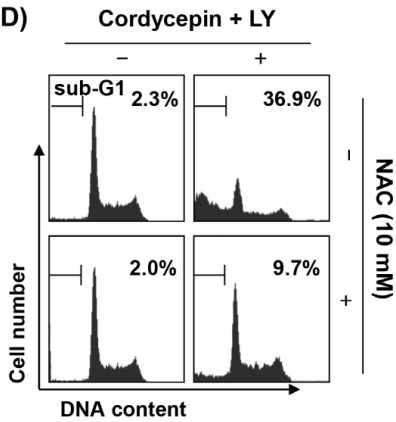

B)

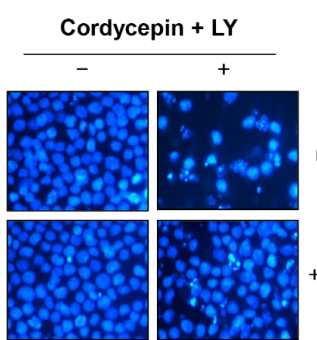

E)

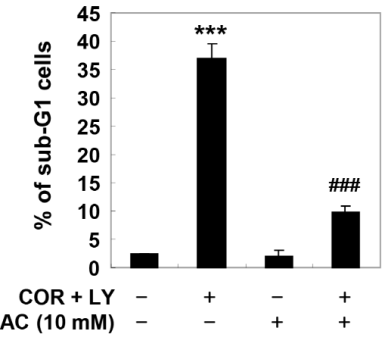

C)

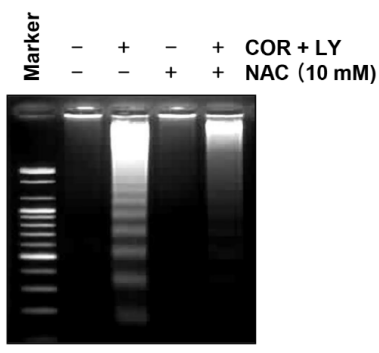

F)

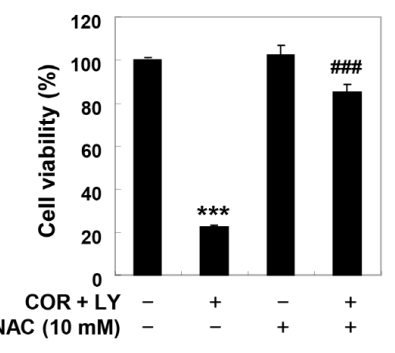

Figure 7. Cordycepin induces the ROS-dependent inactivation of PI3K/Akt pathway in T24 cells. (A) The cells were either treated with $40 \mu \mathrm{g} / \mathrm{mL}$ cordycepin for $48 \mathrm{~h}$, or pre-treated with $10 \mathrm{mM} \mathrm{NAC}$ for $1 \mathrm{~h}$ before $40 \mu \mathrm{g} / \mathrm{mL}$ cordycepin treatment, and then collected. The cellular proteins were prepared, and the expression of PI3K and Akt proteins was evaluated by Western blot analysis. (B-F) The cells were pre-treated with $10 \mu \mathrm{M}$ LY294002 for $1 \mathrm{~h}$, and then treated with $40 \mu \mathrm{g} / \mathrm{mL}$ cordycepin for a further $48 \mathrm{~h}$, in the presence or absence of $10 \mathrm{mM}$ NAC. (B) The DAPI-stained nuclei were then observed by fluorescence microscopy (original magnification, $\times 400$ ). (C) DNA fragmentation was analyzed by the extraction of genomic DNA, electrophoresis in agarose gel, and then visualization by EtBr staining. (D-F) The percentages of apoptotic sub-G1 cells and cell viability were determined by flow cytometry and MTT assay, respectively. (E and F) Data were expressed as the mean $\pm \mathrm{SD}$ of three independent experiments $(* * * p<$ 0.0001 , when compared to control; \#\# $p<0.001$, when compared to cordycepin-treated cells). 
observed in the activated intrinsic pathway $(4,5)$. Cordycepin treatment also significantly increased the activity of caspase-3, and induced the cleavage of PARP. On the other hand, as is well known in previous studies, caspase- 8 activated by the initiation of the extrinsic pathway cleaved and converted $\mathrm{Bid}$, a pro-apoptotic protein belonging to the Bcl-2 family proteins, to tBid $(4,44,45)$. tBid in turn translocates to the mitochondria to promote the permeability of the mitochondrial outer membrane, leading to the accumulation of cytochrome $\mathrm{c}$, counteracting the cytoprotective activity of $\mathrm{Bcl}-2$ protein, and amplifying the intrinsic pathway $(45,46)$. Therefore, the results indicate that cordycepin induced apoptosis in T24 cells by simultaneously activating the extrinsic and intrinsic pathways through tBid-mediated crosstalk.

Abnormal activation of the PI3K/Akt signaling pathway has recently been shown to be involved in the pathogenesis of multiple human tumors, including bladder cancer $(9-11,47,48)$. Activated PI3K initiates the activation of Akt, a downstream kinase of PI3K, which can inhibit apoptosis by protecting caspase cascade through phosphorylation of caspase- 9 , and promotes the expression of anti-apoptotic proteins of the Bcl-2 family proteins, thereby enhancing cell survival and the proliferation of cancer cells $(49,50)$. Because these ultimately contribute to resistance to chemotherapy for inducing apoptosis in cancer cells, PI3K and its regulatory factors are attractive targets for cancer treatment. Therefore, we analyzed whether this signaling pathway was involved in the induction of T24 cell apoptosis by cordycepin, and found cordycepin suppressed the phosphorylated levels of Akt, as well as PI3K. This means that the PI3K/Akt signaling pathway is inactivated by cordycepin treatment, and the results are in good agreement with previous studies performed on several other cancer cell lines (31-36,42,51). Furthermore, in line with our previous study using leukemia cells (36), LY294002, a pharmacological inhibitor of PI3K, significantly enhanced the apoptotic effect of cordycepin and further reduced cell viability, supposing that cordycepin-induced apoptosis is mediated by blocking the PI3K/Akt signaling pathway.

Accumulated evidence has shown that the low levels of ROS in the cell act as a secondary messenger in the intracellular signaling pathways, while excessively high levels of ROS induce oxidation of cellular macromolecules, and promote apoptosis through the activation of extrinsic and/or intrinsic pathways (5254). Recent previous studies have reported that several bioactive compounds generated ROS to activate apoptosis signaling in cancer cells, while ROS-dependent suppressing the activity of the PI3K/Akt signaling pathway (9-11). These observations suggest that inducing the production of ROS in cancer cells can be used in therapeutic strategies, such as the induction of apoptosis through the inhibition of cell survival signals, such as PI3K/Akt. Therefore, we further assessed whether cordycepin-induced apoptosis in T24 cells was correlated with the production of ROS, and the relationship between ROS production and the PI3K/Akt signaling pathway. Consistent with previous studies $(27-28,30,32,41,43)$, our current results showed that cordycepin treatment markedly increased the levels of ROS production; however, as can be predicted, the ROS scavenger, NAC greatly blocked the accumulation of ROS by cordycepin. The quenching of ROS generation also significantly diminished cordycepin-induced disruption of MMP to the control level, followed by significant survival restoration, indicating that ROS act as upstream signaling molecules to enhance cordycepin-induced apoptosis in T24 cells. These results are consistent with our previous findings using prostate cancer cells (43), and signified that the cordycepin-induced apoptosis of T24 cells was ROSdependent. Furthermore, the presence of NAC markedly attenuated cordycepin-induced dephosphorylation of PI3K and Akt proteins, and NAC treatment also significantly blocked the enhanced apoptosis and viability reduction induced by co-treatment of cordycepin and LY294002. Taken together, these results lead us to suggest that the production of ROS by cordycepin plays a critical role in the induction of apoptosis through simultaneous initiation of both extrinsic and intrinsic pathways in T24 cells, and acts as an upstream signal related to the effect of cordycepin on the inactivation of the PI3K/Akt signaling pathway. However, further studies are warranted to determine the direct relationship between cordycepin-mediated inactivation of PI3K/Akt signaling pathway and other cellular signaling pathways, and the identification and role of intracellular organelles involved in ROS generation by cordycepin.

In conclusion, our findings demonstrate that cordycepin exerts an anti-proliferative effect on human bladder cancer T24 cells, through the activation of extrinsic and intrinsic apoptosis pathways. As evidence for this, cordycepin activated caspase- 8 and -9 , which belong to the initiator caspases of the extrinsic and intrinsic pathways, respectively, followed by the activation of effector caspase 3 , resulting in the degradation of PARP. Cordycepin also induced the truncation of Bid and mitochondrial dysfunction, which was associated with an increase in Bax/Bcl-2 expression ratio and cytochrome $\mathrm{c}$ release into the cytoplasm. Moreover, the induction of apoptosis by cordycepin was accompanied by inhibition of the PI3K/Akt signaling pathway, and excessive production of ROS. In addition, artificial interception of the PI3K/Akt signal pathway further increased cordycepin-induced apoptosis, and the interruption of ROS generation led T24 cells to escape from apoptosis, while maintaining the activity of PI3K/Akt signaling pathway. Based on these finding, we suggest that cordycepin has chemopreventive potential by inducing apoptosis through ROS-dependent inactivation of the PI3K / Akt signaling pathway in T24 cells. 


\section{Acknowledgements}

This research was funded by Basic Science Research Program through the National Research Foundation of Korea (NRF) grant funded by the Korea government (2018R1A2B2005705), Republic of Korea.

\section{References}

1. Pfeffer CM, Singh ATK. Apoptosis: A target for anticancer therapy. Int J Mol Sci 2018; 19:448.

2. Hassan M, Watari H, AbuAlmaaty A, Ohba Y, Sakuragi $\mathrm{N}$. Apoptosis and molecular targeting therapy in cancer. Biomed Res Int. 2014; 2014:150845.

3. Kiraz Y, Adan A, Kartal Yandim M, Baran Y. Major apoptotic mechanisms and genes involved in apoptosis. Tumour Biol. 2016; 37:8471-8486.

4. Edlich F. BCL-2 proteins and apoptosis: Recent insights and unknowns. Biochem Biophys Res Commun. 2018; 500:26-34

5. Birkinshaw RW, Czabotar PE. The BCL-2 family of proteins and mitochondrial outer membrane permeabilisation. Semin Cell Dev Biol. 2017; 72:152-162.

6. Karimian A, Mir SM, Parsian H, Refieyan S, MirzaAghazadeh-Attari M, Yousefi B, Majidinia M. Crosstalk between phosphoinositide 3-kinase/Akt signaling pathway with DNA damage response and oxidative stress in cancer. J Cell Biochem. 2019; 120:10248-10272.

7. Yea SS, Fruman DA. Achieving cancer cell death with PI3K/mTOR-targeted therapies. Ann NY Acad Sci. 2013; 1280:15-18.

8. Zhang X, Jin B, Huang C. The PI3K/Akt pathway and its downstream transcriptional factors as targets for chemoprevention. Curr Cancer Drug Targets. 2007; 7:305316.

9. Guo CL, Wang LJ, Zhao Y, Liu H, Li XQ, Jiang B, Luo J, Guo SJ, Wu N, Shi DY. A novel bromophenol derivative BOS-102 induces cell cycle arrest and apoptosis in human A549 lung cancer cells via ROS-mediated PI3K/Akt and the MAPK signaling pathway. Mar Drugs. 2018; 16:E43.

10. Song $X$, Wang Z, Liang H, Zhang W, Ye Y, Li H, Hu Y, Zhang Y, Weng H, Lu J, Wang X, Li M, Liu Y, Gu J. Dioscin induces gallbladder cancer apoptosis by inhibiting ROS-mediated PI3K/AKT signalling. Int J Biol Sci. 2017; 13:782-793.

11. Mi Y, Xiao C, Du Q, Wu W, Qi G, Liu X. Momordin Ic couples apoptosis with autophagy in human hepatoblastoma cancer cells by reactive oxygen species (ROS)-mediated PI3K/Akt and MAPK signaling pathways. Free Radic Biol Med. 2016; 90:230-242.

12. DeGeorge KC, Holt HR, Hodges SC. Bladder cancer: Diagnosis and treatment. Am Fam Physician. 2017; 96:507-514.

13. Janković S, Radosavljević V. Risk factors for bladder cancer. Tumori. 2007; 93:4-12.

14. Stein JP, Lieskovsky G, Cote R, Groshen S, Feng AC, Boyd S, Skinner E, Bochner B, Thangathurai D, Mikhail M, Raghavan D, Skinner DG. Radical cystectomy in the treatment of invasive bladder cancer: Long-term results in 1,054 patients. J Clin Oncol. 2001; 19:666-675.

15. Shi J, Wang Y, Jia Z, Gao Y, Zhao C, Yao Y. Curcumin inhibits bladder cancer progression via regulation of $\beta$-catenin expression. Tumour Biol. 2017; 39:1-8.
16. Kim WJ and Bae SC. Molecular biomarkers in urothelial bladder cancer. Cancer Sci. 2008; 99: 646-652.

17. Kooti W, Servatyari K, Behzadifar M, Asadi-Samani M, Sadeghi F, Nouri B, Zare Marzouni H. Effective medicinal plant in cancer treatment, Part 2: Review study. J Evid Based Complementary Altern Med. 2017; 22:982995.

18. Lou JS, Yao P, Tsim KWK. Cancer treatment by using traditional Chinese medicine: Probing active compounds in anti-multidrug resistance during drug therapy. Curr Med Chem. 2018; 25:5128-5141.

19. Kotecha R, Takami A, Espinoza JL. Dietary phytochemicals and cancer chemoprevention: A review of the clinical evidence. Oncotarget. 2016; 7:52517-52529.

20. Jeong JW, Park C, Cha HJ, Hong SH, Park SH, Kim GY, Kim WJ, Kim CH, Song KS, Choi YH. Cordycepin inhibits lipopolysaccharide-induced cell migration and invasion in human colorectal carcinoma HCT-116 cells through down-regulation of prostaglandin E2 receptor EP4. BMB Rep. 2018; 51:532-537.

21. Yoon SY, Park SJ, Park YJ. The anticancer properties of cordycepin and their underlying mechanisms. Int $\mathrm{J}$ Mol Sci. 2018; 19:E3027.

22. Yue K, Ye M, Zhou Z, Sun W, Lin X. The genus Cordyceps: A chemical and pharmacological review. J Pharm Pharmacol. 2013; 65:474-493.

23. Jin Y, Meng X, Qiu Z, Su Y, Yu P, Qu P. Anti-tumor and anti-metastatic roles of cordycepin, one bioactive compound of Cordyceps militaris. Saudi J Biol Sci. 2018; 25:991-995.

24. Tian X, Li Y, Shen Y, Li Q, Wang Q, Feng, L. Apoptosis and inhibition of proliferation of cancer cells induced by cordycepin. Oncol Lett. 2015; 10:595-599.

25. Tuli HS, Sharma AK, Sandhu SS, Kashyap D. Cordycepin: A bioactive metabolite with therapeutic potential. Life Sci. 2013; 93:863-869.

26. Shao LW, Huang LH, Yan S, Jin JD, Ren SY. Cordycepin induces apoptosis in human liver cancer HepG2 cells through extrinsic and intrinsic signaling pathways. Oncol Lett. 2016; 12:995-1000.

27. Pan BS, Wang YK, Lai MS, Mu YF, Huang BM. Cordycepin induced MA-10 mouse Leydig tumor cell apoptosis by regulating p38 MAPKs and PI3K/AKT signaling pathways. Sci Rep. 2015; 5:13372.

28. Lee HH, Kim SO, Kim GY, Moon SK, Kim WJ, Jeong YK, Yoo YH, Choi YH. Involvement of autophagy in cordycepin-induced apoptosis in human prostate carcinoma LNCaP cells. Environ Toxicol Pharmacol. 2014: 38:239-250.

29. Jeong JW, Jin CY, Park C, Hong SH, Kim GY, Jeong YK, Lee JD, Yoo YH, Choi YH. Induction of apoptosis by cordycepin via reactive oxygen species generation in human leukemia cells. Toxicol In Vitro. 2011; 25:817824.

30. Nasser MI, Masood M, Wei W, Li X, Zhou Y, Liu B, Li J, Li X. Cordycepin induces apoptosis in SGC 7901 cells through mitochondrial extrinsic phosphorylation of PI3K/ Akt by generating ROS. Int J Oncol. 2017; 50:911-919.

31. Wang Y, Lv Y, Liu TS, Yan WD, Chen LY, Li ZH, Piao YS, An RB, Lin ZH, Ren XS. Cordycepin suppresses cell proliferation and migration by targeting CLEC2 in human gastric cancer cells via Akt signaling pathway. Life Sci. 2019; 223:110-119.

32. Bi Y, Li H, Yi D, Bai Y, Zhong S, Liu Q, Chen Y, Zhao G. $\beta$-catenin contributes to cordycepin-induced MGMT 
inhibition and reduction of temozolomide resistance in glioma cells by increasing intracellular reactive oxygen species. Cancer Lett. 2018; 435:66-79.

33. Cui ZY, Park SJ, Jo E, Hwang IH, Lee KB, Kim SW, Kim DJ, Joo JC, Hong SH, Lee MG; Jang IS. Cordycepin induces apoptosis of human ovarian cancer cells by inhibiting CCL5-mediated Akt/NF- $\mathrm{BB}$ signaling pathway. Cell Death Discov. 2018; 4:62.

34. Hueng DY, Hsieh CH, Cheng YC, Tsai WC, Chen Y. Cordycepin inhibits migration of human glioblastoma cells by affecting lysosomal degradation and protein phosphatase activation. J Nutr Biochem. 2017; 41:109116.

35. Baik JS, Mun SW, Kim KS, Park SJ, Yoon HK, Kim DH, Park MK, Kim CH, Lee YC. Apoptotic effects of cordycepin through the extrinsic pathway and $\mathrm{p} 38$ MAPK activation in human glioblastoma U87MG cells. J Microbiol Biotechnol. 2016; 26:309-314.

36. Jang KJ, Kwon GS, Jeong JW, Kim CH, Yoon HM, Kim GY, Shim JH, Moon SK, Kim WJ, Choi YH. Cordyceptin induces apoptosis through repressing hTERT expression and inducing extranuclear export of hTERT. J Biosci Bioeng. 2015; 119:351-357.

37. Ittiudomrak T, Puthong S, Roytrakul S, Chanchao C. $\alpha$-mangostin and apigenin induced cell cycle arrest and programmed cell death in SKOV-3 ovarian cancer cells. Toxicol Res. 2019; 35:167-179.

38. Wei C, Yao X, Jiang Z, Wang Y, Zhang D, Chen X, Fan X, Xie C, Cheng J, Fu J, Leung EL. Cordycepin inhibits drug-resistance non-small cell lung cancer progression by activating AMPK signaling pathway. Pharmacol Res. 2019; 144:79-89.

39. Zhou Y, Guo Z, Meng Q, Lu J, Wang N, Liu H, Liang Q, Quan Y, Wang D, Xie J. Cordycepin affects multiple apoptotic pathways to mediate hepatocellular carcinoma cell death. Anticancer Agents Med Chem. 2017; 17:143149.

40. Liao Y, Ling J, Zhang G, Liu F, Tao S, Han Z, Chen S, Chen Z, Le H. Cordycepin induces cell cycle arrest and apoptosis by inducing DNA damage and up-regulation of p53 in leukemia cells. Cell Cycle. 2015; 14:761-771.

41. Dong J, Li Y, Xiao H, Luo D, Zhang S, Zhu C, Jiang M, Cui M, Lu L, Fan S. Cordycepin sensitizes breast cancer cells toward irradiation through elevating ROS production involving Nrf2. Toxicol Appl Pharmacol.
2019; 364:12-21.

42. Wang D, Zhang Y, Lu J, Wang Y, Wang J, Meng Q, Lee RJ, Wang D, Teng L. Cordycepin, a natural antineoplastic agent, induces apoptosis of breast cancer cells via caspasedependent pathways. Nat Prod Commun. 2016; 11:63-68.

43. Lee HH, Park C, Jeong JW, Kim MJ, Seo MJ, Kang BW, Park JU, Kim GY, Choi BT, Choi YH, Jeong YK. Apoptosis induction of human prostate carcinoma cells by cordycepin through reactive oxygen species mediated mitochondrial death pathway. Int J Oncol. 2013; 42:10361044.

44. Billen LP, Shamas-Din A, Andrews DW. Bid: a Bax-like BH3 protein. Oncogene. 2008; 27:S93-S104.

45. Yin XM. Bid, a BH3-only multi-functional molecule, is at the cross road of life and death. Gene. 2006; 369:7-19.

46. Kantari C, Walczak H. Caspase- 8 and bid: Caught in the act between death receptors and mitochondria. Biochim Biophys Acta. 2011; 1813:558-563.

47. Sathe A, Nawroth R. Targeting the PI3K/AKT/mTOR pathway in bladder cancer. Methods Mol Biol. 2018; 1655: 335-350.

48. Houédé N, Pourquier P. Targeting the genetic alterations of the PI3K-AKT-mTOR pathway: Its potential use in the treatment of bladder cancers. Pharmacol Ther. 2015; 145:1-18.

49. Manning BD, Cantley LC. AKT/PKB signaling: Navigating downstream. Cell. 2007; 129:1261-1274.

50. Song G, Ouyang G, Bao S. The activation of Akt/PKB signaling pathway and cell survival. J Cell Mol Med. 2005; 9:59-71.

51. Cao HL, Liu ZJ, Chang Z. Cordycepin induces apoptosis in human bladder cancer cells via activation of A3 adenosine receptors. Tumour Biol. 2017; 39:1010428317706915.

52. Badrinath N, Yoo SY. Mitochondria in cancer: in the aspects of tumorigenesis and targeted therapy. Carcinogenesis. 2018; 39:1419-1430.

53. Galadari S, Rahman A, Pallichankandy S, Thayyullathil F. Reactive oxygen species and cancer paradox: To promote or to suppress? Free Radic Biol Med. 2017; 104:144-164.

54. Moloney JN, Cotter TG. ROS signalling in the biology of cancer. Semin Cell Dev Biol. 2018; 80:50-64.

(Received August 12, 2019; Revised August 21, 2019; Accepted August 31, 2019) 